\title{
EVALUATION OF ATYPICAL HYPERPLASIA AFTER PERCUTANEOUS VACUUM-ASSISTED BIOPSY OF SUSPICIOUS CALCIFICATIONS
}

Vera Lucia Nunes Aguillar', Giselle Guedes Netto de Mello', Tatiana de Melo Cardoso Tucunduva1, Marcia Mayumi Aracava', Elisandra Cristina Oliveira ${ }^{1}$

${ }^{1}$ Fleury Medicina e Saúde - São Paulo (SP), Brazil.

Introduction: Atypical ductal hyperplasia (ADH), atypical lobular hyperplasia (ALH), lobular carcinoma in situ (LCIS), and flat epithelial atypia (FEA) are part of a heterogeneous group of lesions with uncertain malignant potential and varying rates of malignancy after wide excision. They represent a clinical challenge, given the lack of well-defined approach recommendations. Objective: To determine the local rate of "upgrade" to malignancy (invasive carcinoma or in situ) after wide excision of ADH, ALH, LCIS (classic lobular neoplasia) diagnosed by percutaneous vacuum-assisted biopsy performed only in suspicious calcifications, as well as analyze radiological and histopathological parameters that can be associated with a higher risk of "upgrade". Material and Methods: This is a retrospective analysis of 117 patients diagnosed with ADH, LCIS, and FEA after percutaneous vacuum-assisted biopsy of suspicious calcifications, from 2015 to 2018 . We evaluated radiological parameters - lesion size, morphology of the calcifications, diameter of the needle, and presence of residual calcifications - and histopathological parameters - extension of atypia (focal or multifocal) and association with other atypias. Results: Among the 106 patients included, 77 (73\%) underwent surgery, with a rate of "upgrade" to malignancy of 19.5\% (10 ductal carcinomas in situ, of which 30\% had high grade) and 5 had invasive carcinomas (4 ductal and 1 tubular, all with low grade). In the subgroup analysis, the rate of "upgrade" was $31 \%$ for ADH, $14.7 \%$ for FEA, and 7.7\% for LCIS. Needle diameter $(9 \mathrm{Gx} 11 \mathrm{G})(\mathrm{p}=0.48)$, presence of residual calcifications (less than $90 \%$ of the cluster removed) $(\mathrm{p}=0.73)$, and mean cluster extension (calculated based on the original mammography) ( $p=0.66$ ) showed no statistically significant correlation with an increase in the rate of "upgrade". Amorphous calcifications predominated (60\%), followed by fine pleomorphic ones, with rates of "upgrade" of $11 \%$ and $35 \%$, respectively. Regarding histological parameters, we found no statistically significant difference between groups with focal (up to 2 foci) and multifocal atypia or association with other atypias. Conclusion: Our rate of "upgrade" to malignancy was similar to that of the published literature, and we found no statistically significant radiological or histological criteria for a greater risk of "upgrade". 